\title{
One-dimensional dynamics of magnetic inhomogeneities in a three-layer ferromagnetic structure with different values of the magnetic parameters
}

\author{
E. G. Ekomasov ${ }^{1,2, \dagger}$, R. V. Kudryavtsev ${ }^{2}$, A. M. Gumerov² \\ †EkomasovEG@gmail.com \\ ${ }^{1}$ National Research South Ural State University, 76 Lenin avenue, 454080, Chelyabinsk, Russia \\ ${ }^{2}$ Bashkir State University, 32 Z. Validi St., 450076, Ufa, Russia
}

In this paper, we consider a three-layer ferromagnetic structure, which consists of two broad layers separated by a thin layer. The parameters of magnetic anisotropy, exchange and damping are considered as functions of the coordinate directed along the normal to interfaces between the layers. The case of a point magnetic defect described with the help of Dirac's deltafunction is studied with the values of the parameters of magnetic anisotropy, exchange and damping, which differ from the values of the analogous parameters in the remaining magnet. The dynamics of the domain wall (DW) are studied theoretically with allowance for the excitation of localized magnetization waves in the region of the magnetic defect. Using the collectivecoordinate approach, a system of two equations is obtained for the coordinate of the DW center and the amplitude of the oscillations of the magnetization wave localized in the defect region. From an analysis of this system of equations, it is found how the inhomogeneity of the damping and exchange parameter affects the dynamics of magnetic inhomogeneities. The value of the effective dissipation coefficient is determined, which for the case of motion of the domain wall now becomes dependent on the position of the domain wall. It is shown that accounting for heterogeneity of dissipation and exchange can significantly change the speed and scenario of the dynamics of the DW. The dependence of the minimum value of the magnetic field on the dissipation and exchange inhomogeneity coefficients, at which the DW passes through the defect region, is found.

Keywords: three-layer ferromagnetic structure, dynamics of domain walls and localized magnetic inhomogeneities, sine-Gordon equation, resonant interaction of DW and magnetic soliton.

\section{Одномерная динамика магнитных неоднородностей в трёхслойной ферромагнитной структуре с различными значениями магнитных параметров}

\author{
Екомасов Е. Г. ${ }^{1,2 \dagger}$, Кудрявцев Р. В. ${ }^{2}$, Гумеров А. М. ${ }^{2}$ \\ †EkomasovEG@gmail.com \\ ${ }^{1}$ Южно-Уральский государственный университет (национальный исследовательский университет), \\ пр. Ленина 76, 454080, Челябинск, Россия \\ ${ }^{2}$ Башкирский государственный университет, ул. 3.Валиди 32, 450076, Уфа, Россия
}

В данной работе была рассмотрена трёхслойная ферромагнитная структура, которая состоит из двух широких слоёв, разделённых тонким слоем. Параметры магнитной анизотропии, обмена и затухания считаются функциями от координаты, направленной перпендикулярно границе раздела слоёв. Исследован случай точечного магнитного дефекта, описываемого с помощью дельта-функции Дирака, со значениями параметров магнитной анизотропии, обмена и затухания, отличающимися от значений аналогичных параметров в остальном магнетике. Теоретически изучается динамика доменной границы (ДГ) с учётом возбуждения локализованных волн намагниченности в области магнитного дефекта. С помощью одного из видов теории возмущений для солитонов - коллективно-координатного подхода, который использовался ранее для анализа динамики нелинейных волн уравнения синус-Гордона с примесями, - получена система двух уравнений для координаты центра ДГ и амплитуды колебаний локализованной в области дефекта волны намагниченности. Из анализа этой системы уравнений найдено, как неоднородность па- 
раметра затухания и обмена влияет на динамику магнитных неоднородностей. Определена величина эффективного коэффициента диссипации, который для случая движения доменной границы становится теперь зависящим от положения ДГ. Показано, что учёт неоднородности диссипации и обмена может существенно изменить не только скорость, но и сценарий динамики ДГ (от прохождения через область дефекта до пининга в этой области и резонансного отражения от неё). При некоторых параметрах найдена зависимость минимальной величины магнитного поля от коэффициентов неоднородности диссипации и обмена, при которой ДГ проходит через область дефекта.

Ключевые слова: трёхслойный ферромагнетик, динамика доменных границ и локализованных магнитных неоднородностей, уравнение синус-Гордона, резонансное взаимодействие ДГ и магнитного солитона.

\section{1. Введение}

В последнее время широко исследуются многослойные магнитные структуры в связи с возможностью их практического применения [1]. Часто они представляют собой периодически чередующиеся слои двух материалов с различными физическими свойствами. В настоящее время изучается динамика спиновых волн и магнитных неоднородностей, распространяющихся в таких системах как вдоль, так и перпендикулярно границам раздела слоёв. Во втором случае, часто используются одномерные модели $[2,3]$. Отметим, что зачастую именно изучение одномерных моделей позволяет понять влияние тех или иных магнитных параметров на рассматриваемый процесс (см., например, [4-5]).

При исследовании динамики линейных и нелинейных волн намагниченности, распространяющихся перпендикулярно слоям, существует два подхода. В первом из них, используемом часто для изучения динамики спиновых волн, для описания динамики намагниченности в слое рассматривается уравнение Ландау-Лифшица с постоянными параметрами материала, а на границе слоёв требуется выполнение определённых граничных условий [6]. Во втором подходе, наличие слоёв, отличающихся друг от друга значением одного или нескольких магнитных параметров, учитывается пространственной модуляцией магнитных параметров материала (см., например, $[7,8]$ ).

Влияние локальной и периодической одномерной пространственной модуляции магнитных параметров материала (ПММП) на характер распространения, спектр и затухание спиновых волн и на высокочастотные свойства изучено достаточно хорошо (см., например, [9]). При определённых условиях, изучение одномерной динамики магнитных неоднородностей приводит к интересной и с математической точки зрения задаче нахождения решения уравнения типа синус-Гордона с переменными коэффициентами, имеющего важное значение для многих областей современной физики [10]. В связи со сложностью задачи, исследователями рассматривалась, как правило, модуляция лишь отдельных параметров магнитной системы. Часто учитывалась, например, модуляция магнитной анизотропии для случая двух-, трёх- и пятислойного магнетика, причём задачи изучались как аналитическими, так и численными методами, как для точечных, так и для протяжённых дефектов $[4,7,8,11-13]$. В настоящей работе проведено аналитическое исследование динамики доменных границ в трёхслойной ферромагнитной структуре с учётом одновременной пространственной модуляции параметров магнитной анизотропии, обмена и затухания.

\section{2. Основные уравнения и результаты}

Рассмотрим трёхслойную ферромагнитную структуру, состоящую из двух широких одинаковых слоёв, разделённых тонким слоем с изменёнными значениями параметра анизотропии, обмена и затухания. Параметры анизотропии, обмена и затухания считаем теперь функциями от координаты $x$, направленной перпендикулярно границе раздела слоёв, т.е. в системе имеется один магнитный «дефект». Будем далее изучать динамику расположенных в плоскости $y z$ локализованных магнитных неоднородностей. Обычно, при решении динамических задач удобно перейти к сферическим координатам вектора намагниченности $\mathbf{M}(\cos \varphi \sin \theta, \sin \varphi, \cos \varphi \cos \theta)$, где $0 \leq \theta \leq 2 \pi-$ угол в плоскости $y z$ между направлением вектора магнитного момента и осью лёгкого намагничивания (ось $O z),-\pi / 2<\varphi<\pi / 2-$ угол, описывающий выход М из плоскости доменной границы (ДГ). Учитывая в плотности энергии магнетика обменное взаимодействие и анизотропию, и считая $\varphi \ll 1$ [1], уравнение движения для намагниченности в угловых переменных можно представить в следующем обезразмеренном виде [12]:

$$
\begin{aligned}
u_{t t}-u_{x x} & +\sin u=2 h \sin u / 2+\varepsilon \delta(x) \sin u- \\
& -\gamma \delta(x) u_{x x}-\gamma \delta_{x}(x) u_{x}-\alpha u_{t}+\alpha \beta \delta(x) u_{t},
\end{aligned}
$$

где $u=2 \theta, \delta(x)$ - дельта-функция Дирака, $\varepsilon, \gamma, \beta-$ константы, определяющие изменение параметров анизотропии, обмена и затухания на точечном дефекте, $h$ - величина внешнего магнитного поля, направленного вдоль оси $z, \alpha-$ значение параметра затухания в области вне дефекта. Уравнение (1) - модифицированное уравнение синус-Гордона (МУСГ). Заметим также, что уравнение вида (1) можно получить и для случая двухподрешёточных ферримагнетиков и слабых ферромагнетиков [14].

Рассмотрим структуру и динамику ДГ с учетом возможности возбуждения в области дефекта локализованных волн намагниченности. Будем считать, что $\varepsilon, \gamma, h$, $\alpha \ll 1$, а слагаемые в правой части уравнения (1) малы. Применим приближенный коллективно-координатный подход, уже использованный ранее (см., например, [12 - 13]). Первая коллективная переменная $X=X(t)$ обозначает координату центра ДГ. Учитываем далее наличие локализованной волны намагниченности в области дефекта (или примесной моды) с помощью введения второй коллективной переменной $a=a(t)-$ амплитуды локализованной волны. Выражение для описания динамической структуры ДГ и локализованной волны намагниченности будем брать в виде, аналогичном использованному ранее [12-13]: 


$$
\begin{gathered}
u_{0}=4 \operatorname{arctg}\left(\mathrm{e}^{x-X(t)}\right), \\
u_{1}=a(t) \mathrm{e}^{-\varepsilon|x| / 2} .
\end{gathered}
$$

В приближении малых по амплитуде локализованных колебаний, можно считать, что

$$
a(t)=a_{0} \cos \left(\Omega_{\text {single }} t+\theta_{0}\right),
$$

где $\theta_{0}-$ начальная фаза. Решая уравнение (1) для случая одиночного дефекта и при отсутствии ДГ, можно получить для частоты примесной моды следующее выражение:

$$
\Omega_{\text {single }}=\sqrt{1-\varepsilon^{2} / 4}
$$

Общее решение задачи $u_{\text {ansatz }}$ будем далее искать в виде:

$$
u_{\text {ansatz }}=u_{0}+u_{1}=4 \operatorname{arctg}\left(e^{x-X(t)}\right)+a(t) e^{-\varepsilon|x| / 2} .
$$

Считаем далее, что $a(t)$ достаточно мало (является величиной порядка малого $\varepsilon$ ). Тогда, в рамках рассматриваемого приближения имеем, что $u_{1} \ll 1$. Применение метода коллективных переменных (см. $[7,10])$ с использованием разложения нелинейного слагаемого в лагранжиане задачи в ряд Тейлора до членов второго порядка по $\varepsilon$ :

$$
\cos u_{\text {ansatz }}=\cos \left(u_{0}+u_{1}\right) \approx \cos u_{0}-u_{1} \sin u_{0}-\frac{u_{1}^{2}}{2} \cos u_{0}
$$

и отбрасыванием малых слагаемых приводит после интегрирования к новому эффективному лагранжиану, зависящему уже от новых переменных $X$ и $а$. Уравнения движения для $X(t)$ и $a(t)$ можно получить, подставив полученный эффективный лагранжиан в систему уравнений Лагранжа второго рода:

$$
\begin{aligned}
4 \ddot{X}(t) & +4 \alpha\left(1-\frac{\beta}{2 \cosh ^{2}(X(t))}\right) \dot{X}(t)+4 h+ \\
& +2(\varepsilon+\gamma) \frac{\sinh (X(t))}{\cosh ^{3}(X(t))}+\varepsilon \frac{\sinh ^{2}(X(t))-1}{\cosh ^{3}(X(t))} a(t)- \\
& -\varepsilon \frac{\sinh (X(t))}{\cosh ^{3}(X(t))} a^{2}(t)+\frac{\alpha \beta \dot{a}(t)}{\cosh (X(t))} a(t)=0, \\
\ddot{a}(t)+ & \alpha\left(1-\frac{\varepsilon \beta}{2}\right) \dot{a}(t)+\left(1-\frac{\varepsilon^{2}}{2}+\frac{\varepsilon^{2}}{\cosh ^{2}(X(t))}\right) a(t)- \\
& -\varepsilon^{2} \frac{\sinh ^{2}(X(t))}{\cosh ^{2}(X(t))}+\frac{\alpha \beta \varepsilon \dot{X}(t)}{\cosh (X(t))}=0 .
\end{aligned}
$$

Система уравнений (7) - уже система обыкновенных дифференциальных уравнений второго порядка. Первое уравнение системы (7) описывает динамику ДГ с учетом наличия дефекта и локализованной магнитной неоднородности. Второе уравнение системы (7) описывает динамику локализованной магнитной неоднородности с учетом наличия дефекта и ДГ.

Рассмотрим далее, как неоднородность затухания, не рассмотренная ранее, влияет на динамику магнитных неоднородностей. Например, для локализованных волн намагниченности при отсутствии затухания и положении ДГ вдали от дефекта $(X(t) \rightarrow \infty)$ имеем из (7) случай гармонических колебаний для амплитуды с частотой равной (4). При учете $\alpha>0$ эти колебания затухают с коэффициентом затухания $\alpha(1-\varepsilon \beta / 2)$, т.е. положительный $\beta$ ослабляет затухание амплитуды колебаний, отрицательный - усиливает (рис. 1).
Для случая движения доменной границы эффективный коэффициент диссипации становится зависящим от положения ДГ и равным $4 \alpha\left(1-\beta /\left(2 \operatorname{ch}^{2} X(t)\right)\right)$. Так как из физических соображений следует, что параметр затухания всегда должен быть больше нуля, то $\beta<2$. При положительном значении параметра $\beta$ затухание уменьшается, а при отрицательном - увеличивается (рис. 2). Из рис. 2 видно, что учет неоднородности диссипации может существенно изменить не только скорость, но и сценарий динамики ДГ (от прохождения через область дефекта (рис. $2 \mathrm{~b}, 1)$ до пининга в этой области

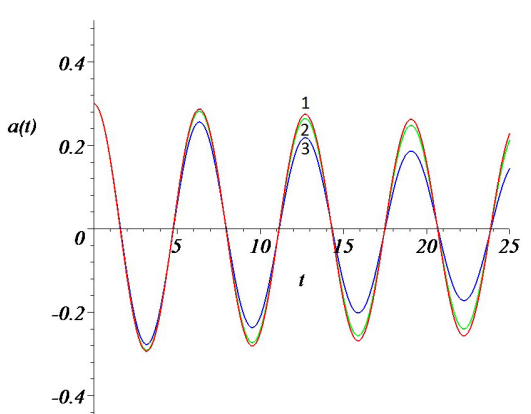

Рис. 1. Зависимость амплитуды локализованной волны намагниченности от времени $a(t)$, рассчитанная в отсутствии ДГ при $a(0)=0,3, \partial a(0) / \partial t=0, \varepsilon=0,3, h=0, \alpha=0,02, \gamma=0: 1) \beta=2$, 2) $\beta=0,3) \beta=-10$.

Fig. 1. Dependence of the amplitude of the localized magnetization wave on time $a(t)$, calculated in the absence of DW at $a(0)=0,3$, $\partial a(0) / \partial t=0, \varepsilon=0,3, h=0, \alpha=0,02, \gamma=0: 1) \beta=2,2) \beta=0,3) \beta=-10$.
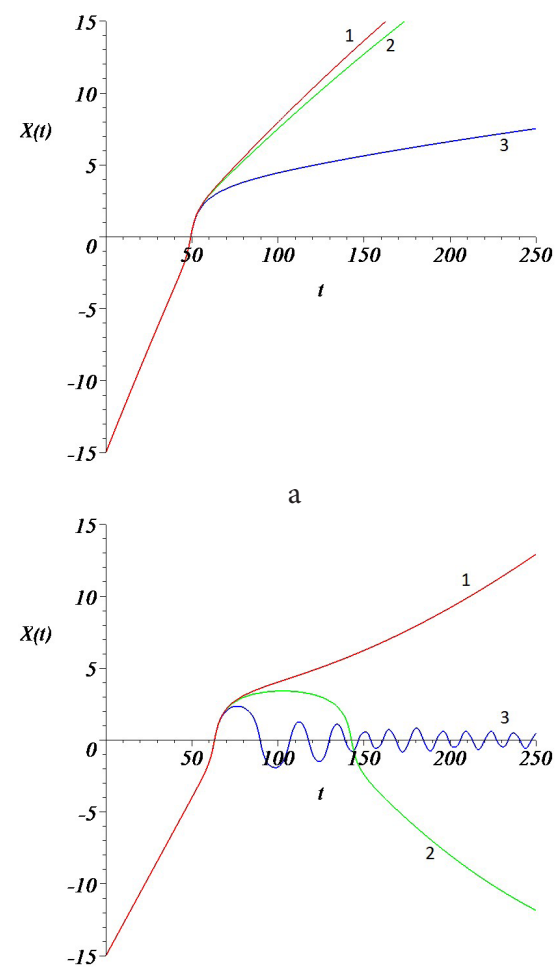

b

Рис. 2. Зависимость координаты центра ДГ $X(t)$ от времени, рассчитанная при $X(0)=-15, \quad \varepsilon=0,7, \quad \alpha=0,002, \quad \gamma=0$, (a) $\partial X(0) / \partial t=0,3, \quad h=0, \quad$ (b) $\partial X(0) / \partial t=0,22, \quad h=-0,00044 \quad$ и: 1) $\beta=2$,2) $\beta=0,3) \beta=-10$.

Fig. 2. Dependence of the coordinate of the center of the DW $X(t)$ on time, calculated at $X(0)=-15, \varepsilon=0,7, \alpha=0,002, \gamma=0$, (a) $\partial X(0) / \partial t=0,3, h=0, \quad$ (b) $\partial X(0) / \partial t=0,22, h=-0,00044$ and: 1) $\beta=2,2) \beta=0,3) \beta=-10$. 
(рис. 2b, 3) и резонансного отражения от нее (рис. 2b, 2)). Это связано с тем, что в уравнение для ДГ добавляется слагаемое $\alpha \beta(\partial a(t) / \partial t) / \operatorname{ch}(X(t))$, зависящее от скорости колебаний амплитуды локализованной волны и положения ДГ. Аналогично, в уравнение для локализованной магнитной неоднородности добавляется слагаемое $\varepsilon \alpha \beta(\partial X(t) / \partial t) / \operatorname{ch}(X(t))$, зависящее от скорости и положения ДГ. Резонансное отражение от притягивающего потенциала связано в данном случае, как и раньше [12], с резонансным обменом при определённой скорости энергией между ДГ и локализованной магнитной неоднородностью.

Найдём минимальную величину магнитного поля $h_{\kappa p}$, при которой ДГ проходит через область дефекта с первого раза (связанную в данном случае с коэрцитивной силой) при $X(0)=-15, \varepsilon=0,7, \alpha=0,002, \gamma=0, \partial X(0) / \partial t=-h / \alpha$ и различных значениях $\beta$ :

Табл. 1. Зависимость $h_{\kappa p}$ от $\beta$.

Table 1. Dependence of $h_{c r}$ on $\beta$.

\begin{tabular}{|c|c|c|c|c|c|c|c|c|}
\hline$-\beta$ & -2 & 0 & 2 & 4 & 6 & 8 & 10 & 12 \\
\hline$-h_{c r}, 10^{-5}$ & 44 & 45 & 46 & 48 & 49 & 50 & 52 & 53 \\
\hline$-\beta$ & 14 & 16 & 18 & 20 & 22 & 24 & 26 & \\
\hline$-h_{c r}, 10^{-5}$ & 54 & 56 & 57 & 58 & 59 & 60 & 61 & \\
\hline
\end{tabular}

Как видно из таблицы, зависимость $h_{\kappa p}$ от $\beta$ в данной модели при исследованных параметрах в рамках принятой точности носит линейный характер. При уменьшении $\beta$, т. е. при росте затухания в области дефекта, величина $h_{\kappa р}$ увеличивается.

Параметр $\gamma$, описывающий отличие параметра обмена в области дефекта, присутствует для наших приближений в уравнениях (7) только в одном слагаемом. Оно увеличивает силу притяжения ДГ дефектом, что увеличивает его скорость. Увеличение скорости ДГ приводит к увеличению диссипации и степени возбуждения примесной моды, так что скорость ДГ после прохождения дефекта снижается (см. рис. 3a). Из рис. $3 \mathrm{~b}$ видно, что учет неоднородности обмена может существенно изменить не только скорость, но и сценарий динамики ДГ (от прохождения через область дефекта (рис. 3b, 1), до пининга в этой области (рис. $3 \mathrm{~b}, 3)$ и резонансного отражения от неё (рис. 3b, 2).

Найдём минимальную величину магнитного поля $h_{\kappa p}$, при которой ДГ проходит через область дефекта с первого раза при $X(0)=-15, \varepsilon=0,7, \alpha=0,002, \beta=0$, $\partial X(0) / \partial t=-h / \alpha$ и различных значениях $\gamma$ :

Табл. 2. Зависимость $h_{\kappa p}$ от $\gamma$.

Table 2. Dependence of $h_{c r}$ on $\gamma$.

\begin{tabular}{|c|c|c|c|c|c|c|}
\hline$\gamma$ & 0 & 0,1 & 0,2 & 0,3 & 0,4 & 0,5 \\
\hline$h_{c r},-10^{-5}$ & 45 & 49 & 52 & 55 & 57 & 59 \\
\hline$\gamma$ & 0,6 & 0,7 & 0,8 & 0,9 & 1 & \\
\hline$h_{c r},-10^{-5}$ & 60 & 61 & 62 & 63 & 64 & \\
\hline
\end{tabular}

Из таблицы видно, что при увеличении $\gamma$ величина $h_{\kappa p}$ при данных параметрах увеличивается с замедлением практически в полтора раза.

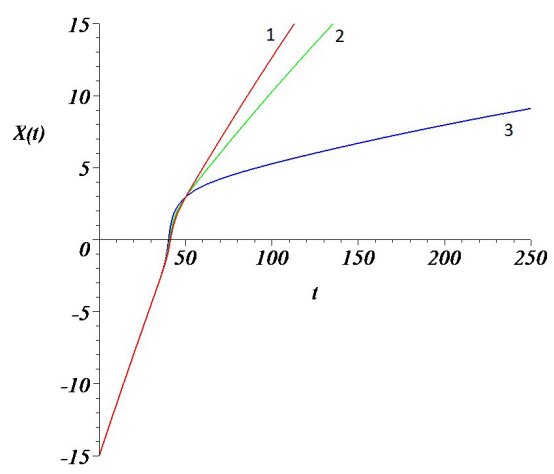

a

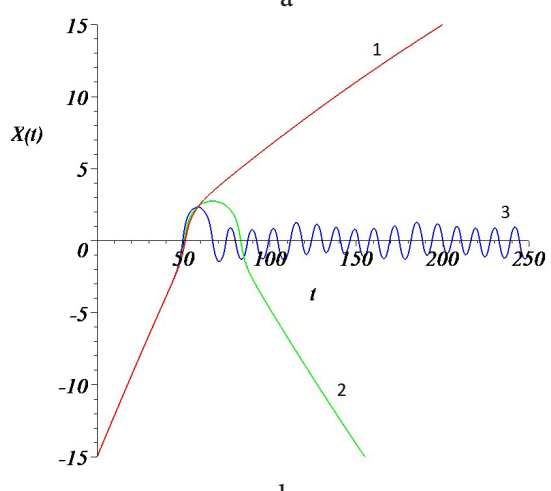

Рис. 3. Зависимость координаты центра ДГ $X(t)$ от времени, рассчитанная при $X(0)=-15, \varepsilon=0,7, h=0, \alpha=0,002, \beta=0$, (а) $\partial X(0) / \partial t=0,36$, (b) $\partial X(0) / \partial t=0,29$, и 1) $\gamma=0$, 2) $\gamma=0,3,3) \gamma=1$.

Fig. 3. Dependence of the coordinate of the center of the DW $X(t)$ on time, calculated at $X(0)=-15, \varepsilon=0,7, h=0, \alpha=0,002, \beta=0$, (a) $\partial X(0) / \partial t=0,36$, (b) $\partial X(0) / \partial t=0,29$, and 1) $\gamma=0$, 2) $\gamma=0,3,3) \gamma=1$.

\section{3. Заключение}

Для случая трёхслойной ферромагнитной структуры изучена динамика доменной границы с учётом возбуждения локализованных волн намагниченности в области тонкого магнитного слоя. С помощью коллективно-координатного подхода получена система двух уравнений для координаты центра ДГ и амплитуды колебаний локализованной в области тонкого слоя волны намагниченности. Определена величина эффективного коэффициента диссипации, который для случая движения доменной границы становится теперь зависящим от положения ДГ. Показано, что учёт неоднородности диссипации и обмена может существенно изменить не только скорость, но и сценарий динамики ДГ (от прохождения через область дефекта до пининга в этой области и резонансного отражения от неё). Найдена зависимость минимальной величины внешнего магнитного поля от коэффициентов неоднородности диссипации и обмена, при которой ДГ проходит через область магнитного дефекта. Можно предположить, что при дальнейших исследованиях подобные результаты будут получены и при учёте конечного размера тонкого магнитного слоя.

Благодарность/Acknowledgements. Статья выполнена при поддержке Правительства РФ (Постановление № 211 om 16.03.2013 г.), соглашение № 02. A03.21.0011. 


\section{Литература/References}

1. Denny D. Tang, Yuan-Jen Le. Magnetic Memory Fundamentals and Technolog. Cambridge, Cambridge University Press, New York. (2010) 196 p.

2. A.B. Borisov, V.V. Kiselev. Quasi-one-dimensional magnetic solitons. Moscow, FIZMATLIT. (2014) 520 p. (in Russian) [А.Б. Борисов, В.В. Киселёв. Квазиодномерные магнитные солитоны. Москва, ФИЗМАТЛИТ. 2014. 520 с.]

3. E. Della Torre, C. M. Perlov. J. Appl. Phys. 69, 4596 (1991).

4. V.N. Nazarov, R.R. Shafeev, M. A. Shamsutdinov, I. Yu Lomakina. Phys. Solid State. 54, 298 (2012). (in Russian) [В.Н. Назаров, Р.Р. Шафеев, М.А. Шамсутдинов, И. Ю. Ломакина. ФТТ. 54 (2), 282 (2012).]

5. V. V. Kiselev, A. A. Rascovalov. Chaos, Solitons \& Fractals. 45, 1551 (2012).

6. V.V. Kruglyak, A.N. Kuchko, V.I. Finokhin. Phys. Solid State. 46, 867 (2004). (in Russian) [В. В. Кругляк, А. Н. Кучко, В. И. Финохин. ФТТ. 46 (5), 842 (2004).]

7. E.G. Ekomasov, A.M. Gumerov, R.R. Murtazin, R.V. Kudryavtsev, A.E. Ekomasov, N.N. Abakumova. Solid state phenomena. 233-234, 51 - 54 (2015).

8. E. G. Ekomasov, R. R. Murtazin, V. N. Nazarov. Journal of
Magnetism and Magnetic Materials. 385, 217 (2015).

9. V.A. Ignatchenko, Yu.I. Mankov, A.A. Maradudin. Phys. Rev. B. 62 (3), 2181 (2000).

10. J. Cuevas-Maraver, P.G. Kevrekidis, F. Williams (Eds.). The Sine-Gordon Model and Its Applications: From Pendula and Josephson Junctions to Gravity and Highenergy Physics, volume 10. Springer. (2014) 263 p.

11. E.G. Ekomasov, R.R. Murtazin, O.B. Bogomazova, A. M. Gumerov. JMMM. 339, 133 (2013).

12. E.G. Ekomasov, A.M. Gumerov. Letters on materials. 4 (4), 237-240 (2014). (in Russian) [А. М. Гумеров, Е. Г. Екомасов. Письма о материалах. 3 (2), $103-105$ (2013).]

13. E.G. Ekomasov, A.M. Gumerov, R.V. Kudryavtsev. Letters on materials. 6 (2), 138 - 140 (2016). (in Russian) [Е. Г. Екомасов, А. М. Гумеров, Р. В. Кудрявцев. Письма о материалах. 6 (2), 138 - 140 (2016).]

14. M. A. Shamsutdinov, V. N. Nazarov, I. Yu. Lomakina, and others. Ferro- and antiferromagnetodynamics. Nonlinear oscillations, waves and solitons. Moscow, Science. (2009) 368 p. (in Russian) [M. А. Шамсутдинов, В. Н. Назаров, И.Ю. Ломакина и др. Ферро- и антиферромагнитодинамика. Нелинейные колебания, волны и солитоны. Москва, Наука. 2009. 368 с.]. 